\title{
HECKE ALGEBRA CHARACTERS AND IMMANANT CONJECTURES
}

\author{
MARK HAIMAN
}

\section{INTRODUCTION}

The main purpose of this article is to announce and provide supporting evidence for two conjectures about the characters of the Hecke algebra $H_{n}(q)$ of type $A_{n-1}$, evaluated at elements of its Kazhdan-Lusztig basis. In addition, we prove a conjectured immanant inequality for Jacobi-Trudi matrices (definitions below) and show how our conjectures would imply stronger inequalities of a similar kind.

The immanant inequalities belong to the combinatorial theory of symmetric functions and consequently have gained considerable attention in algebraic combinatorics since their introduction by Goulden and Jackson [9]; see [10, 29, 30]. The Hecke algebra conjectures presented here are, however, independent of the application which led to their discovery, and because of their striking and unexpected nature, they should be of interest to a broader audience. In particular, they appear to reflect aspects of the geometry of the flag variety that cannot yet be understood using available geometric machinery. It has also been discovered that Hecke algebras of type $A_{n-1}$ arise naturally in the study of knots [7, 14], quantum groups [13], and Von Neumann algebras [15, 34]. Their character theory, in particular, plays an important role, via the Ocneanu trace and the commutant relationship between $H_{n}(q)$ and the quantum group $U G L_{n}(q)$. Thus there are important reasons to seek a better understanding of the characters.

The first of our conjectures asserts that certain virtual characters, i.e., integral linear combinations of irreducible characters, take values on the KazhdanLusztig basis which are polynomials in $q$ with nonnegative, symmetric, and unimodal integer coefficients. A corresponding assertion for the irreducible characters follows from the theory of intersection homology and perverse sheaves for Schubert varieties [3, 27], together with the fact that the Kazhdan-Lusztig cell representations [17] are irreducible for type $A_{n-1}$. This fact is a weaker statement than our conjecture, however, since the irreducible characters are nonnegative combinations of the virtual characters we consider. In fact, the

Received by the editors March 5, 1992.

1991 Mathematics Subject Classification. Primary 14M15; Secondary 05E05.

Research supported in part by N.S.F. grants DMS-8717795 and DMS-9119355.

(C) 1993 American Mathematical Society $0894-0347 / 93 \$ 1.00+\$ .25$ per page 
conjecture for these virtual characters is best possible: any virtual character for which the assertion holds must be a nonnegative combination of these.

The second conjecture asserts that, for purposes of character evaluation, most Kazhdan-Lusztig basis elements are reducible to sums of certain of the simplest possible ones. To be precise, we pick out certain permutations, called codominant, whose corresponding Schubert varieties are smooth and very simple to describe. Consequently, the corresponding Kazhdan-Lusztig basis elements also have an extremely simple description. The conjecture then states that for each Kazhdan-Lusztig basis element $C_{w}^{\prime}$ there is a sum $C_{w_{1}}^{\prime}+\cdots+C_{w_{k}}^{\prime}$ of basis elements with $w_{i}$ codominant, such that

$$
\chi\left(C_{w}^{\prime}\right)=\chi\left(C_{w_{1}}^{\prime}+\cdots+C_{w_{k}}^{\prime}\right)
$$

for every Hecke algebra character $\chi$. In particular, the second conjecture would reduce the first conjecture to its special case for codominant elements, thus eliminating Kazhdan-Lusztig polynomials from the problem.

We will make clear in our later discussion why there are good reasons to expect deep connections between these conjectures and the geometry of the flag variety. Unfortunately, even the powerful machinery of perverse sheaves and intersection homology appears, at least for the moment, to provide inadequate information for a solution of the conjectures.

We are able to prove various special cases of the conjectures. Several authors $[19,24,33]$ have considered the problem of evaluating characters of $H_{n}(q)$ at elements of its natural basis, a problem which is the $q$-analog of determining the character table of the symmetric group $S_{n}$. We have made use of these results in order to prove a special case of our first conjecture in $\S 4$. It is conceivable that a similar approach could be used to resolve the first conjecture for codominant elements. To do so, one would have to prove a $q$-analog of Proposition 5.1, without the restriction assumed there on the codominant permutation $w_{f}$. The problem of evaluating characters at arbitrary elements of the Kazhdan-Lusztig basis is in general much more difficult since little explicit information is known about the Kazhdan-Lusztig polynomials, which appear as the coefficients expressing this basis in terms of the natural one.

In addition to the two aforementioned conjectures, this paper contains some theorems, whose relevance we now explain, along with the overall organization of the paper.

The present investigation originated with the author's discovery that Kazhdan-Lusztig theory for the Hecke algebra $H_{n}(q)$ is the natural setting for the immanant inequalities conjectured by Goulden and Jackson [9] and extended by Stembridge, Stanley, and Greene [10, 29, 30]. It turns out that all these immanant inequalities would follow from our first Hecke algebra conjecture, combined with a theorem proved here (Theorem 1.5) relating a generating function for immanants to the Kazhdan-Lusztig basis for $H_{n}(q)$. Even without the Hecke algebra conjecture, Theorem 1.5 implies an immanant inequality stronger than the one proved by Greene. 
Since many readers will not be familiar with the subject of immanant inequalities (or even the word 'immanant'), we give a brief historical review of the topic in $\S 1$ and explain how our results and conjectures apply. As the main theorem of the section, Theorem 1.5, has a rather technical proof, using the Kazhdan-Lusztig conjecture on composition series of Verma modules, we defer its proof to the end of the paper, in $\S 7$.

We then turn to the presentation of our two central conjectures and some general remarks about them, followed by sections dealing with computational and special case evidence for the conjectures with $q$ general, with $q=1$, and with $q=0$, respectively. If desired, the paper may be read beginning with $\S 2$, except for the definition (1.2) of monomial characters, since the Hecke algebra conjectures and related results do not depend on the application to immanants.

We have included an appendix covering definitions and results from Kazhdan-Lusztig theory, most of which are standard by now, but which may not be familiar to all readers, especially from the combinatorial audience. Otherwise unexplained notation and facts stated without citation refer either to the appendix, or, where symmetric functions and Young tableaux are concerned, to the book of Macdonald [23].

\section{IMMANANTS}

We begin with the definition of immanants and a review of conjectured inequalities involving them. A much more complete survey may be found in [30].

Let $\chi$ be an irreducible character of $S_{n}$, or more generally any class function, and let $A$ be an $n \times n$ matrix. Littlewood's immanants [22] are matrix functionals generalizing the determinant and the permanent, defined by

$$
\operatorname{Imm}_{\chi}(A)=\sum_{w \in S_{n}} \chi(w) a_{1, w(1)} \cdots a_{n, w(n)} .
$$

When $\chi=\chi_{\lambda}$ is the irreducible character indexed by the partition $\lambda$ of $n$, we abbreviate $\operatorname{Imm}_{\chi_{\lambda}}(A)$ to $\operatorname{Imm}_{\lambda}(A)$. In particular, the determinant and the permanent are the immanants $\operatorname{Imm}_{\left(1^{n}\right)}(A)$ and $\operatorname{Imm}_{(n)}(A)$ corresponding to the sign character and the trivial character, respectively.

The rule of thumb for immanant inequalities is that if a matrix has nonnegative minors in some suitable sense, then its irreducible immanants, or certain linear combinations of them, ought to be nonnegative.

The oldest branch of the subject, going back to Schur, involves immanants of positive definite Hermitian matrices, typified by the 'Schur dominance theorem' and 'Lieb permanental dominance conjecture', which state the following.

Theorem 1.1 (Schur [25]). If $A$ is positive definite, then $\operatorname{Imm}_{\lambda}(A)-f_{\lambda} \operatorname{det}(A) \geq$ 0 for all $\lambda$.

Conjecture 1.1 (Lieb [21]). If $A$ is positive definite, then $f_{\lambda} \operatorname{per}(A)-\operatorname{Imm}_{\lambda}(A)$ $\geq 0$. 
In each of these statements $f_{\lambda}=\chi_{\lambda}(1)$ is the degree of the irreducible character $\chi_{\lambda}$.

The more recent branches concern immanants of totally positive matrices ( $A$ is totally positive if every minor of $A$ is nonnegative) and Jacobi-Trudi matrices (defined below). In their strongest forms the conjectured inequalities concern monomial immanants, which we now define.

From the theory of symmetric functions, the irreducible characters of $S_{n}$ are given by $\chi_{\lambda}(w)=\left\langle s_{\lambda}, p_{\tau(w)}\right\rangle$, where $s_{\lambda}$ denotes a Schur function, $p_{\tau(w)}$ is the power-sum symmetric function indexed by the partition $\tau(w)$ corresponding to the decomposition of $w$ into cycles, and $\left\langle s_{\lambda}, s_{\mu}\right\rangle=\left\langle m_{\lambda}, h_{\mu}\right\rangle=\delta_{\lambda \mu}$. Here $h_{\mu}$ and $m_{\lambda}$ denote the complete and the monomial symmetric functions, respectively.

Thus we have a natural linear correspondence mapping the Schur functions of degree $n$ to the irreducible characters of $S_{n}$. Under this correspondence the monomial symmetric function $m_{\lambda}$ is carried to a 'monomial' virtual character $\phi_{\lambda}$ given by

$$
\phi_{\lambda}(w)=\left\langle m_{\lambda}, p_{\tau(w)}\right\rangle .
$$

The corresponding monomial immanants are the immanants $\operatorname{Imm}_{\phi_{\lambda}}(A)$. Since the Schur functions are nonnegative linear combinations of monomial symmetric functions, nonnegativity statements for monomial immanants are in general stronger than their counterparts for irreducible immanants.

For totally positive matrices we have the following analogs of Schur dominance and permanental dominance. Actually, Conjecture 1.2 is a stronger statement which implies both permanental dominance and Theorem 1.2. No correspondingly strong statement holds for positive definite matrices.

Theorem 1.2 (Stembridge [32]). If $A$ is totally positive, then $\operatorname{Imm}_{\lambda}(A)-$ $f_{\lambda} \operatorname{det}(A) \geq 0$ for all $\lambda$.

Conjecture 1.2 (Stembridge [32]). If $A$ is totally positive, then $\operatorname{Imm}_{\phi_{\lambda}}(A) \geq 0$ for all $\lambda$.

Our main concern will be the third and most combinatorial branch of the subject, namely, immanant inequalities for Jacobi-Trudi matrices. Let $h_{n}$ denote the complete homogeneous symmetric function of degree $n$ in countably many variables $X=\left(x_{1}, x_{2}, \ldots\right)$. By convention, $h_{n}=0$ for $n<0$ and $h_{0}=1$. Let $\mu$ and $\nu$ be partitions with $n$ parts, written in descending order as $\left(\mu_{1} \geq \mu_{2} \geq \cdots \geq \mu_{n}\right)$. We allow 0 as a part. Jacobi-Trudi matrices are of the form

$$
H_{\mu / \nu}(X)=\left[h_{\mu_{i}-\nu_{j}}(X)\right]_{i, j=1}^{n} .
$$

The classical Jacobi-Trudi matrix would be $H_{\mu+\delta / \nu+\delta}$ in this notation, where $\delta=(n-1, \ldots, 1,0)$. The Jacobi-Trudi identity expresses the (skew) Schur 
functions in terms of homogeneous symmetric functions as

$$
s_{\mu / \nu}=\operatorname{det}\left(H_{\mu+\delta / \nu+\delta}\right) \text {. }
$$

We have altered the notation in order to include the possibility that $H_{\mu / \nu}$ may have repeated rows or columns, which is a relevant case for immanants other than the determinant.

Since the minors of a Jacobi-Trudi matrix are skew Schur functions, they are 'nonnegative' in the sense either of being nonnegative linear combinations of monomial symmetric functions or of Schur functions.

In their survey of applications of immanants to matrices having combinatorial significance, Goulden and Jackson [9] conjectured the following result, which has since been proved by Greene.

Theorem 1.3 (Greene [10]). The irreducible immanants $\operatorname{Imm}_{\lambda}\left(H_{\mu / \nu}\right)$ of JacobiTrudi matrices are nonnegative linear combinations of monomial symmetric functions for all $\lambda$.

This theorem can be strengthened in either or both of two ways: we can ask for nonnegativity in terms of Schur functions rather than monomials, or we can pass to monomial immanants. The three conjectures so obtained are due to Stembridge [30]. Our main application of Kazhdan-Lusztig theory to immanants is the proof of one of these conjectures. Thus we state the first conjecture as a theorem and the other two as conjectures, as follows. Note that Conjecture 1.4 implies both of the others.

Theorem 1.4. If $H_{\mu / \nu}$ is a Jacobi-Trudi matrix, then $\operatorname{Imm}_{\lambda}\left(H_{\mu / \nu}\right)-f_{\lambda} \operatorname{det}\left(H_{\mu / \nu}\right)$ is a nonnegative linear combination of Schur functions.

Conjecture 1.3 (Stembridge [30]). The monomial immanants $\operatorname{Imm}_{\phi_{\lambda}}\left(H_{\mu / \nu}\right)$ of Jacobi-Trudi matrices are non-negative linear combinations of monomial symmetric functions for all $\lambda$.

Conjecture 1.4 (Stembridge [30]). The monomial immanants $\operatorname{Imm}_{\phi_{\lambda}}\left(H_{\mu / \nu}\right)$ of Jacobi-Trudi matrices are nonnegative linear combinations of Schur functions for all $\lambda$.

To conclude this section, we shall explain how Theorem 1.4 can be proven using Kazhdan-Lusztig theory and how Conjectures 1.2-1.4 would follow from the conjecture on Hecke algebra characters developed in the next section.

It is convenient to introduce the following formal sum, which serves as a kind of generating function for the immanants of $H_{\mu / \nu}$. Let

$$
I_{\mu / \nu}=\sum_{w \in S_{n}} T_{w} h_{\mu-w(\nu)}(X) .
$$

This is an element of $H_{n}(1) \otimes \Lambda(X)$, the group algebra of $S_{n}$ with coefficients extended to the ring of symmetric functions $\Lambda(X)$. Extending $S_{n}$ characters $\chi$ in the obvious way to $\Lambda(X)$-linear functionals on $H_{n}(1) \otimes \Lambda(X)$, we may 
express the immanants of Jacobi-Trudi matrices as

$$
\operatorname{Imm}_{\chi}\left(H_{\mu / \nu}(X)\right)=\chi\left(I_{\mu / \nu}\right) .
$$

With this notation, Theorem 1.4 is an immediate consequence of the following two statements.

Theorem 1.5. $I_{\mu / \nu}$ is a nonnegative linear combination of the basis elements $C_{w}^{\prime}(1) s_{\kappa}(X)$ for $H_{n}(1) \otimes \Lambda(X)$, where $\left\{C_{w}^{\prime}(q)\right\}$ is the Kazhdan-Lusztig basis of the Hecke algebra $H_{n}(q)$.

Lemma 1.1. Let $\chi_{\lambda}$ be an irreducible character of $H_{n}(q)$, of degree $f_{\lambda}$, and let $C_{w}^{\prime}$ be a Kazhdan-Lusztig basis element. Put $\chi=\chi_{\lambda}-f_{\lambda} \chi_{\left(1^{n}\right)}$. Then $\chi\left(q^{\ell(w) / 2} C_{w}^{\prime}\right)$ is a polynomial with nonnegative integer coefficients. In fact, these coefficients are unimodal and symmetric about $q^{\ell(w) / 2}$.

Theorem 1.5 is proven in $\S 7$; the coefficients which appear have an explicit interpretation as multiplicities of irreducible composition factors in a certain infinite-dimensional tensor product of $\mathfrak{s l}_{n}$ modules.

Let us now prove Lemma 1.1 .

Proof. First of all, the character $\chi_{\left(1^{n}\right)}$, which is the sign representation of $H_{n}(q)$, vanishes on $C_{w}^{\prime}$ unless $w=1$, because the Kazhdan-Lusztig polynomials $P_{v, w}$ obey $P_{v, w}=P_{s v, w}$ for any simple reflection $s$ such that $s w<s$. Since $C_{1}^{\prime}=T_{1}=1$, we have $\chi_{\lambda}\left(C_{1}^{\prime}\right)=f_{\lambda}$ and $\chi_{\left(1^{n}\right)}\left(C_{1}^{\prime}\right)=1$. Thus $\chi\left(C_{w}^{\prime}\right)$ is zero if $w=1$ and we may replace $\chi$ with $\chi_{\lambda}$ otherwise.

Recall that the irreducible $H_{n}(q)$ module $V_{\lambda}$ can be constructed as a cell representation: a quotient $E_{\lambda} / F_{\lambda}$ of submodules $F_{\lambda} \subseteq E_{\lambda} \subseteq H_{n}(q)$, where each of $E_{\lambda}$ and $F_{\lambda}$ is the linear span of a subset of the Kazhdan-Lusztig basis $\left\{C_{w}^{\prime}\right\}$. Thus $V_{\lambda}$ has a Kazhdan-Lusztig basis given by those elements $C_{u}^{\prime}$ which are in $E_{\lambda}$ but not $F_{\lambda}$, and $a \in H_{n}(q)$ acts by a matrix whose $(u, v)$ entry is the coefficient of $C_{u}^{\prime}$ in $a C_{v}^{\prime}$. In particular, when $a=q^{\ell(w) / 2} C_{w}^{\prime}$ the diagonal entries (whose sum is the trace $\chi_{\lambda}\left(q^{\ell(w) / 2} C_{w}^{\prime}\right)$ ) are the structure coefficients $q^{\ell(w) / 2} f_{w, u}^{u}(q)$, which are polynomials with the required nonnegativity, symmetry, and unimodality properties.

In the same way that Theorem 1.4 follows from Theorem 1.5 and Lemma 1.1 , it is clear that Conjecture 1.4 (and the weaker Conjecture 1.3) would follow from Theorem 1.5 and a stronger version of Lemma 1.1 in which monomial characters replace the characters $\chi$. Such a strengthening of Lemma 1.1 is precisely our first Hecke algebra conjecture, Conjecture 2.1.

In fact, not only the conjectures on Jacobi-Trudi matrices but also Conjecture 1.2 on totally positive matrices would follow from Conjecture 2.1 -see the remarks at the end of the next section. 


\section{A FIRST CONJECTURE}

Since the irreducible characters of $H_{n}(q)$ correspond to those of $\mathbb{C} S_{n}=$ $H_{n}(1)$, the same is true of virtual characters. In particular, the monomial characters $\phi_{\lambda}$ of $S_{n}$ defined by (1.2) have unique analogs for $H_{n}(q)$, specializing to (1.2) for $q=1$. Our first conjecture, which is natural to make in view of the proof of Theorem 1.4 via Theorem 1.5 and Lemma 1.1, concerns evaluations of these monomial characters.

Conjecture 2.1. For every monomial character $\phi_{\lambda}$ of $H_{n}(q)$ and every KazhdanLusztig basis element $C_{w}^{\prime}, \phi_{\lambda}\left(q^{\ell(w) / 2} C_{w}^{\prime}\right)$ is a polynomial with nonnegative integer coefficients. Moreover, these coefficients are unimodal and symmetric about $q^{\ell(w) / 2}$.

Note that the symmetry of the coefficients about $q^{\ell(w) / 2}$ holds for every virtual character. This follows from Lemma 1.1, or, to give a more elementary reason, from the invariance of $C_{w}^{\prime}$ under the Hecke algebra involution defined in (8.5).

We shall discuss four broad aspects of Conjecture 2.1 here. More detailed information can be found with the supporting evidence in $\S \$ 4-6$.

Geometry. By definition, the homogeneous symmetric function $h_{n}$ is the $\operatorname{sum} \sum_{|\lambda|=n} m_{\lambda}$ of all monomials. Hence the sum $\sum_{|\lambda|=n} \phi_{\lambda}$ is the character corresponding to $h_{n}=s_{(n)}$, i.e., the 'trivial' character $\chi_{(n)}\left(T_{w}\right)=q^{w}$. Applying this to $C_{w}^{\prime}$ we obtain

$$
\sum_{|\lambda|=n} \phi_{\lambda}\left(q^{\ell(w) / 2} C_{w}^{\prime}\right)=\sum_{v \leq w} P_{v, w}(q) q^{v} .
$$

The expression on the right is the Poincare series for the global intersection homology of the Schubert variety $\Gamma_{w}$ associated to $w$.

The intersection homology decomposition theorem of [3] gives, for a projective algebraic map $f: Y \rightarrow X$ of complex projective varieties, a natural direct sum decomposition of the intersection homology of $Y$. The Poincare series of the summands have the symmetry and unimodality properties we want for $\phi_{\lambda}\left(q^{\ell(w) / 2} C_{w}^{\prime}\right)$. It is to be expected that (2.1) reflects some such decomposition, where $Y$ is either the Schubert variety $\Gamma_{w}$ or some related variety.

Best possible statement. Conjectures 1.2 and 1.4 are best possible, in that by appropriate choices of totally positive matrix $A$ or Jacobi-Trudi matrix $H_{\mu / \nu}$, one can see that any immanant with the required nonnegativity property must be a nonnegative linear combination of monomial immanants. It follows that any virtual character with the properties asserted for irreducible characters in Lemma 1.1 and for monomial characters in Conjecture 2.1 is necessarily a nonnegative linear combination of monomial characters. This can also be seen from the evaluation of $\phi_{\lambda}\left(q^{\ell(w) / 2} C_{w}^{\prime}\right)$ given by Proposition 4.1 for $w$ the longest element of a parabolic subgroup of $S_{n}$. 
Total positivity. Stembridge [32] proves Theorem 1.2 by associating to a matrix $A$ an element $[A]$ of the group algebra $\mathbb{C} S_{n}$, in a manner analogous to the definition (1.5) of $I_{\mu / \nu}$. The main part of his proof is a demonstration that, for totally positive $A,[A]$ is a nonnegative linear combination of certain group algebra elements on which the irreducible characters take nonnegative values. In fact, if we let $\sum H$ denote the sum of all elements in a subgroup $H \subseteq S_{n}$, his proof shows that $[A]$ is a sum of products of elements of the form $\sum W_{J}$ for various parabolic subgroups $W_{J}$. It follows that $[A]$ is a nonnegative linear combination of Kazhdan-Lusztig basis elements $C_{w}^{\prime}(1)$, and therefore Conjecture 2.1 implies Conjecture 1.2.

Other Coxeter groups. There is probably an analog of Conjecture 2.1 for other Coxeter groups or at least Weyl groups. At present, however, it is not apparent what the analog of a monomial character should be. Nor do we even know that the analog of Lemma 1.1 for irreducible characters holds, since in general the cell representations are reducible.

\section{A SECOND CONJECTURE}

Whereas Conjecture 2.1 appears natural in view of the proof of Theorem 1.4 , our second conjecture arose completely unexpectedly from examining data computed to verify Conjecture 2.1. The conjecture applies to all characters and could in principle have been discovered independently of any computation of monomial characters. It is a bit of good fortune, however, that because Conjecture 2.1 is best possible, tables of monomial characters are full of zeroes and small, highly recognizable polynomials, making linear relationships stand out. This, together with the fact that the codominant permutations defined below seem to play a special role in immanant inequalities, is what made it possible to detect the second conjecture by 'inspection'.

To state the conjecture, we must define a certain class of permutations which we call codominant because they are of the form $w_{0} v$, where $v$ is one of Lascoux's dominant vexillary permutations [20] and $w_{0}$ is the longest permutation in $S_{n}$.

Definition. Let $f(1) \leq f(2) \leq \cdots \leq f(n)$ be a nondecreasing sequence of integers with $f(n)=n$ and $f(i) \geq i$ for all $i$. Let $w_{f}$ be the lexicographically greatest permutation satisfying $w_{f}(i) \leq f(i)$ for all $i$. Such a permutation $w_{f}$ is called codominant.

Proposition 3.1. Let $w_{f}$ be codominant. Then $w_{f}$ determines $f$ by the rule $f(i)=\max \left\{w_{f}(j) \mid j \leq i\right\}$. We have $\{v \mid v(i) \leq f(i) \forall i\}=\left\{v \mid v \leq w_{f}\right\}$, where $\leq$ is Bruhat order. The permutations $v \leq w_{f}$ may be described as those whose permutation matrices are zero in a pattern of entries forming a Ferrers diagram in the lower-left corner of the matrix. With respect to a fixed base flag $0 \subset F_{1} \subset F_{2} \subset \cdots \subset F_{n}=\mathbb{C}^{n}$ in the flag variety $\mathscr{F}_{n}$, the Schubert variety $\Gamma_{w_{f}}$ consists of all flags $0 \subset G_{1} \subset G_{2} \subset \cdots \subset G_{n}=\mathbb{C}^{n}$ satisfying $G_{i} \subseteq F_{f(i)}$ for all $i$. 
This variety is smooth. In particular, $P_{v, w_{f}}=1$ for all $v \leq w_{f}$, or equivalently

$$
q^{\ell\left(w_{f}\right) / 2} C_{w_{f}}^{\prime}=\sum_{v \leq w_{f}} T_{v} .
$$

Proof. Clearly $w_{f}(1), w_{f}(2), \ldots$ can be obtained writing down in turn for each $i$ the greatest number between 1 and $f(i)$ which has not yet been used. In particular, for $i$ such that $f(i-1)<f(i)$ (and for $i=1$ ) we have $w_{f}(i)=$ $f(i)$. The rule for recovering $f$ follows immediately.

Suppose $v(i) \leq f(i)$ for all $i$, suppose $v(j)>v(k)$ for some $j<k$, and let $v^{\prime}=v \cdot(j k)$. Then clearly $v^{\prime}(i) \leq f(i)$ for all $i$. This shows that the set $\{v \mid v(i) \leq f(i) \forall i\}$ is closed going downward in Bruhat order and hence contains $\left\{v \mid v \leq w_{f}\right\}$.

Again suppose $v(i) \leq f(i)$ for all $i$, and suppose $v \neq w_{f}$. Let $j$ be the least index for which $v(j) \neq w_{f}(j)$. Let $k=v^{-1}\left(w_{f}(j)\right)$, so $v(k)=w_{f}(j)$. Since $v(i)=w_{f}(i) \neq w_{f}(j)$ for $i<j$, we have $k>j$. Since $w_{f}$ is lexicographically greater than $v, v(j)<w_{f}(j)=v(k)$. Hence $v^{\prime}=v \cdot(j k)>v$ in Bruhat order. Again we have $v^{\prime}(i) \leq f(i)$ for all $i$. We may repeatedly replace $v$ by $v^{\prime}$ until we reach $w_{f}$, showing $v \leq w_{f}$ and proving $\{v \mid v(i) \leq f(i) \forall i\}=\{v \mid$ $\left.v \leq w_{f}\right\}$.

The permutation matrix description merely restates the condition $v(i) \leq f(i)$ for all $i$.

The description of $\Gamma_{w_{f}}$ is a direct translation of the condition $v(i) \leq f(i)$ for all $i$ into Schubert conditions on the flag. Consider the variety $\Gamma^{k}$ of partial flags $0 \subset G_{1} \subset G_{2} \subset \cdots \subset G_{k}$ with $\operatorname{dim}\left(G_{i}\right)=i$ satisfying $G_{i} \subseteq F_{f(i)}$ for all $i$. We see that $\Gamma^{k+1}$ is a bundle over $\Gamma_{k}$ with fiber $\mathbb{P}^{f(k+1)-k-1}(\mathbb{C})$. Hence each $\Gamma^{k}$ is smooth, including $\Gamma^{n}=\Gamma_{w_{f}}$. The remaining statements follow immediately.

We remark that $w$ is codominant if and only if there are no $i<j<k$ with $w(j)<w(k)<w(i)$. The number of these in $S_{n}$ is well known to be the Catalan number $C_{n}=\frac{1}{n+1}\left(\begin{array}{c}2 n \\ n\end{array}\right)$.

Now we may state our conjecture.

Conjecture 3.1. Write $a \sim b$ if $a, b \in H_{n}(q)$ are such that $\chi(a)=\chi(b)$ for every Hecke algebra character $\chi$. Then for every $w \in S_{n}$ there exist codominant permutations $w_{1}, \ldots, w_{k}$ such that $C_{w}^{\prime} \sim C_{w_{1}}^{\prime}+\cdots+C_{w_{k}}^{\prime}$. The number $k$ of these is given by $P_{1, w}(1)$, and more precisely,

$$
P_{1, w}(q)=\sum_{i=1}^{k} q^{\left(\ell(w)-\ell\left(w_{i}\right)\right) / 2} .
$$


Proof of (3.2), assuming what goes before. Consider the character $\chi$ corresponding to the action of $H_{n}(q)$ on the complex vector space with basis consisting of the points of the finite 'flag variety' over $\mathrm{GL}_{n}(q)$, for $q$ a prime power. We have $\chi\left(T_{1}\right)=[n]_{q} !=1(1+q) \cdots\left(1+q+\cdots+q^{n-1}\right)$ and $\chi\left(T_{v}\right)=0$ for $v \neq 1$. Hence $a \sim b$ implies that $a$ and $b$ contain $T_{1}$ with the same coefficient. Applying this to $q^{\ell(w) / 2} C_{w}^{\prime} \sim \sum_{i} q^{\left(\ell(w)-\ell\left(w_{i}\right)\right) / 2} q^{\ell\left(w_{i}\right) / 2} C_{w_{i}}^{\prime}$, using $P_{1, w_{i}}=1$, yields (3.2).

We have several remarks to make about Conjecture 3.1.

Reduction. If Conjecture 3.1 holds, it clearly reduces Conjecture 2.1 to the case that $w$ is codominant.

Geometry. Like Conjecture 2.1, Conjecture 3.1 seems to suggest some sort of direct sum decomposition of perverse sheaf theoretic objects. However, the picture is much murkier. For Conjecture 2.1, one can envision the kind of setup that might account for the character values, even if it is hard to guess exactly what varieties and maps are involved. It is not at all evident how to account for the decomposition given by Conjecture 3.1. Even how to give a geometric interpretation to the relation $\sim$ is far from clear. Our feeling is that Conjecture 3.1 reflects deeper phenomena than Conjecture 2.1.

In particular, there are many smooth Schubert varieties corresponding to noncodominant permutations. Yet the conjecture suggests that each such variety should be 'equivalent' in some undetermined character theoretic sense to a codominant one. In particular, its cohomology ring must have the same Poincare series as that of a codominant one. It seems to the author rather amazing that anything like this should be so.

Combinatorics of Kazhdan-Lusztig polynomials. Any rule for explicitly listing the codominant permutations $w_{1}, \ldots, w_{k}$ provided by the conjecture affords a combinatorial interpretation of the coefficients of $P_{1, w}$ for all $w$. Instances of the conjecture may be computed to seek clues to such a rule. Unfortunately the resulting information is incomplete, since the expressions $C_{w}^{\prime} \sim C_{w_{1}}^{\prime}+\cdots+C_{w_{k}}^{\prime}$ are in general not unique.

Since $P_{1, w}(0)=1$, the conjectured expansion $C_{w}^{\prime} \sim C_{w_{1}}^{\prime}+\cdots+C_{w_{k}}^{\prime}$ must have a unique 'leading term', which we may take to be $C_{w_{1}}^{\prime}$, with $\ell\left(w_{1}\right)=$ $\ell(w)$. It seems likely that any rule for the expansion will obey certain additional conditions (which still do not determine it uniquely): (1) if $w$ is codominant, then $w_{1}=w$; (2) more generally, if $w \leq w^{\prime}$ with $w^{\prime}$ codominant, then $w_{1} \leq w^{\prime}$; and (3) $w_{i} \leq w_{1}$ for all $i$.

One might expect the leading $w_{1}$ to depend on $w$ in a Bruhat-order-preserving fashion, but that is incompatible with (1). Consider $w=3412$ in $S_{4}$. For this $w, w_{1}$ must be 3241 or 2431 . But 3214 and 1432 are codominants less than $w$ and neither candidate for $w_{1}$ is greater than both of these.

More immanant conjectures. Stanley and Stembridge [29] offered conjectures which reduce Conjecture 1.4 to special cases associated with codominant 
permutations. Restated in the language used here, their [29, Conjecture 5.1] becomes:

Conjecture 3.2 [29]. Define $E_{\mu / \nu}^{\kappa} \in H_{n}(1)$ by expanding $I_{\mu / \nu}$ with respect to Schur functions:

$$
I_{\mu / \nu}=\sum_{|\kappa|=|\mu|-|\nu|} E_{\mu / \nu}^{\kappa} S_{\kappa}(X) .
$$

Then there exist codominant permutations $w_{1}, \ldots, w_{k}$ such that $E_{\mu / \nu}^{\kappa} \sim$ $C_{w_{1}}^{\prime}(1)+\cdots+C_{w_{k}}^{\prime}(1)$.

A futher conjecture in the same paper [29, Conjecture 5.4] refines the above statement with some conditions relating the terms in the expansion $C_{w_{1}}^{\prime}(1)+$ $\cdots+C_{w_{k}}^{\prime}(1)$ to Young tableaux.

Conjecture 3.2 would follow from Conjecture 3.1, since by Theorem 1.5, $E_{\mu / \nu}^{\kappa}$ is a nonnegative linear combination of Kazhdan-Lusztig basis elements. One of the conditions which the more refined conjecture [29, Conjecture 5.4] imposes on the terms is that each $w_{i} \leq w_{f}$, where $w_{f}$ is the largest codominant avoiding the pattern of zeros in the Jacobi-Trudi matrix $H_{\mu / \nu}$. This would follow from the conditions proposed above on the rule for the expansion $C_{w}^{\prime} \sim$ $C_{w_{1}}^{\prime}+\cdots+C_{w_{k}}^{\prime}$. We do not see how to account for the other conditions in their conjecture.

\section{THE GENERIC CASE}

In this section we discuss special cases of the conjectures which hold when $q$ is regarded as an indeterminate.

Both Conjectures 2.1 and 3.1 have been verified by computer for $n<7$. Table 1 on the following page gives the monomial character values $\phi_{\lambda}\left(q^{\ell(w) / 2} C_{w}^{\prime}\right)$ and expansions $C_{w}^{\prime} \sim C_{w_{1}}^{\prime}+C_{w_{2}}^{\prime}$ with $w_{i}$ codominant for $w \in S_{4}$. Note that $a \sim b$ is equivalent to $\phi_{\lambda}(a)=\phi_{\lambda}(b)$ for all $\lambda$. Thus equivalences such as $C_{1243}^{\prime} \sim C_{1324}^{\prime} \sim C_{2134}^{\prime}$ (showing that the expansion by codominants is nonunique) can be read off at once from the table.

In the following proposition we collect those evaluations $\phi_{\lambda}\left(q^{\ell(w) / 2} C_{w}^{\prime}\right)$ which can be obtained readily from well-known properties of cell representations and Hecke algebra characters.

Proposition 4.1. (1) Let $W_{J}=S_{\lambda_{1}} \times \cdots \times S_{\lambda_{k}}$ be a parabolic subgroup of $W=$ $S_{n}$. Let $w_{J} \in W_{J}$ be its longest element. Then

$$
\phi_{\mu}\left(q^{\ell\left(w_{J}\right) / 2} C_{w_{J}}^{\prime}\right)= \begin{cases}\sum_{w \in W_{J}} q^{\ell(w)}=\left[\lambda_{1}\right]_{q} ! \cdots\left[\lambda_{k}\right]_{q} !, & \mu=\lambda \\ 0, & \mu \neq \lambda .\end{cases}
$$




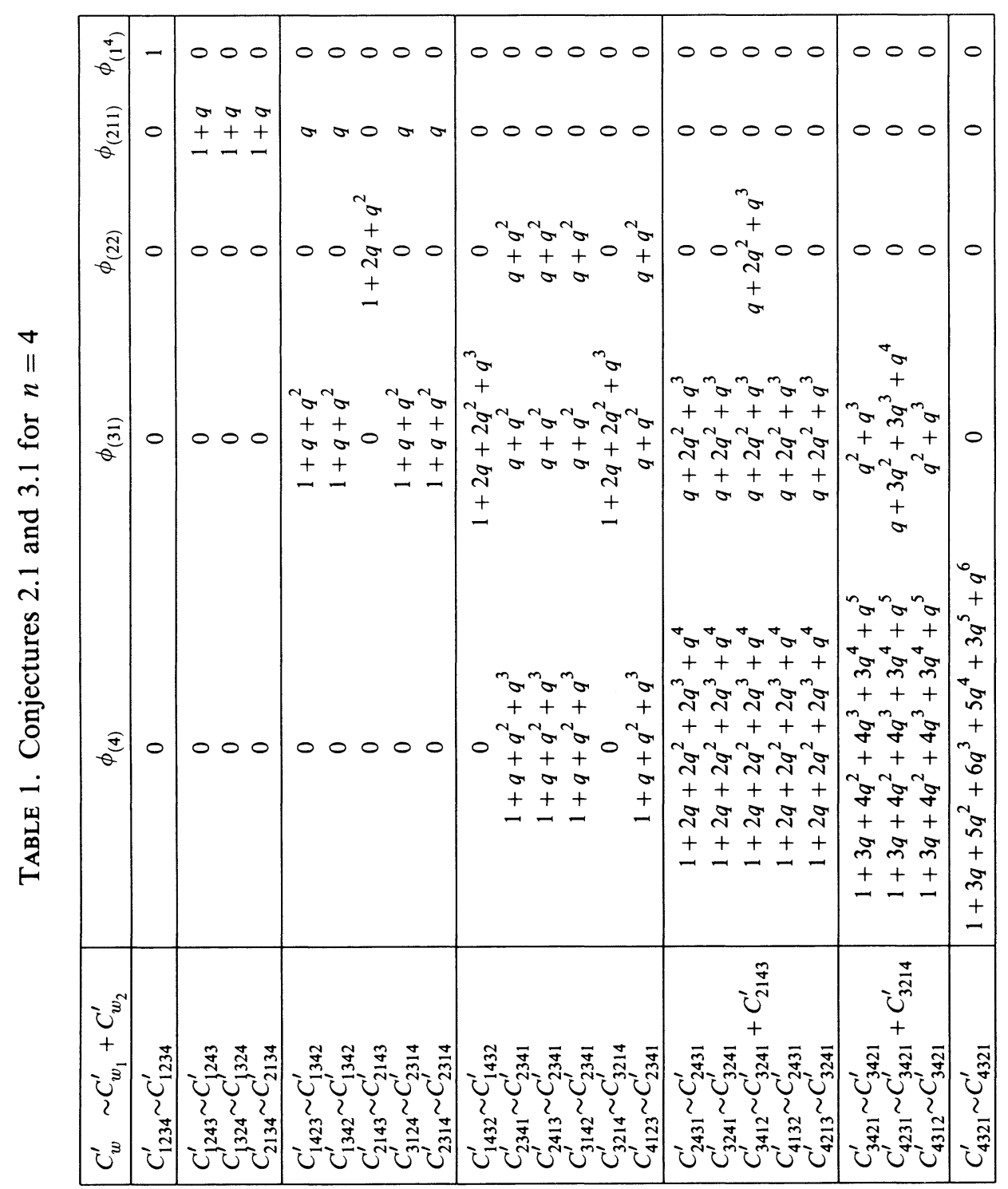


(2) Let $w$ be an arbitrary element of $W_{J}=S_{\lambda_{1}} \times \cdots \times S_{\lambda_{k}}$, and write it as $w=w_{1} w_{2} \cdots w_{k}$ with $w_{i} \in S_{\lambda_{i}}$. Then

$$
\phi_{\mu}\left(q^{\ell(w) / 2} C_{w}^{\prime}\right)=\sum_{\substack{\mu^{(1)} \cup \ldots \cup \mu^{(k)} \in \mu \\\left|\mu^{(i)}\right|=\lambda_{i}}} \prod_{i=1}^{k} \phi_{\mu^{(i)}}\left(q^{\ell\left(w_{i}\right) / 2} C_{w_{i}}^{\prime}\right) .
$$

(3) Let $\lambda$ be the shape of the tableaux $P(w), Q(w)$ associated with $w$ by the Schensted correspondence, and $\lambda^{\prime}$ the conjugate partition. Then $\phi_{\mu}\left(q^{\ell(w) / 2} C_{w}^{\prime}\right)=$ 0 unless $\mu \geq \lambda^{\prime}$ in dominance order.

Proof. We begin with (2). Formula (4.2) follows from the fact that $q^{\ell(w) / 2} C_{w}^{\prime}=$ $q^{\ell\left(w_{1}\right) / 2} C_{w_{1}}^{\prime} \cdots q^{\ell\left(w_{k}\right) / 2} C_{w_{k}}^{\prime}$, together with the rule for restriction of $H_{n}(q)$ characters to $H_{\lambda_{1}}(q) \otimes \cdots \otimes H_{\lambda_{k}}(q)$ (the branching coefficients do not involve $q$ and so are the same as for $S_{n}$ characters).

Given (2), (1) reduces to the case $W_{J}=S_{n}, w_{J}=w_{0}$. The single element $C_{w_{0}}^{\prime}$ spans a two-sided ideal in $H_{n}(q)$, the Kazhdan-Lusztig cell affording the 'trivial' one-dimensional representation $V_{(n)}$ in which $T_{w}$ maps to $q^{\ell(w)}$. Hence $C_{w_{0}}^{\prime}$ acts as 0 in every cell representation except $V_{(n)}$, where it acts as $\sum_{w \in S_{n}} q^{\ell(w)}=[n]_{q} !$. This is (4.1) for $W_{J}=S_{n}$.

For (3), recall that the Kazhdan-Lusztig cells for $S_{n}$ are given by the Schensted correspondence, so that $C_{w}^{\prime}$ belongs to the two-sided cell indexed by the partition $\lambda^{\prime}$. Since the ordering $<_{L R}$ among two-sided cells is opposite to dominance order among partitions, the two-sided ideal $H C_{w}^{\prime} H$ in $H=H_{n}(q)$ is spanned by elements $C_{v}^{\prime}$ belonging to cells indexed by $\mu \geq \lambda^{\prime}$. In particular, $C_{w}^{\prime}$ acts as 0 in the cell representation $V_{\mu}$ unless $\mu \geq \lambda^{\prime}$. Since the monomial characters and the irreducible characters are dominance-lower-triangular with respect to one another, this implies (3).

Next we shall prove Conjecture 2.1 in the case $w=s_{1} \cdots s_{k}$, where $s_{1}, \ldots$, $s_{k}$ are distinct simple reflections (i.e., adjacent transpostions in $S_{n}$ ). By (4.2) we may assume that $s_{1}, \ldots, s_{k}$ are all the simple reflections.

Proposition 4.2. Let

$$
\psi(a)=\sum_{|\lambda|=n} \phi_{\lambda}(a) h_{\lambda}(X)=\sum_{|\lambda|=n} \chi_{\lambda}(a) s_{\lambda}(X)
$$

for any $a \in H_{n}(q)$. Let $w_{n}$ be a Coxeter element of $S_{n}$, that is, the product of all $n-1$ simple reflections, in any order. Then $\psi\left(q^{(n-1) / 2} C_{w_{n}}^{\prime}\right)$ depends only on $n$, and we have the generating function

$$
1+\sum_{n \geq 1} \psi\left(q^{(n-1) / 2} C_{w_{n}}^{\prime}\right)=\frac{\sum_{n \geq 0} h_{n}(X)}{1-q \sum_{n \geq 2}\left(1+q+\cdots+q^{n-2}\right) h_{n}(X)} .
$$


Proof. Write $w_{n}$ as $s_{1} \cdots s_{n-1}$. The elements $v \leq w_{n}$ in Bruhat order correspond to subsets $I=\left\{i_{1}<\cdots<i_{j}\right\} \subseteq\{1, \ldots, n-1\}$, with $v=s_{i_{1}} \cdots s_{i_{j}}$. For all such $v, P_{v, w_{n}}=1$, or

$$
q^{(n-1) / 2} C_{w_{n}}^{\prime}=\sum_{I} T_{s_{i_{1}} \cdots s_{i_{j}}}
$$

This follows by induction from the formula (8.8) for $C_{s}^{\prime} C_{w}^{\prime}$.

Ram [24] has evaluated $\psi\left(T_{v}\right)$ for $T_{v}$ of the form appearing in (4.5), obtaining

$$
\psi\left(T_{s_{i_{1}} \cdots s_{i_{j}}}\right)=\frac{q^{n}}{(q-1)^{\ell(\mu)}} h_{\mu}\left(\left(1-q^{-1}\right) X\right) .
$$

Here $\mu=\left(j_{1}, j_{2}-j_{1}, \ldots, n-j_{k}\right)$ is the composition of $n$ determined by $\left\{j_{1}<\cdots<j_{k}\right\}=\{1, \ldots, n-1\} \backslash I$; equivalently it describes the cycle structure of the permutation $s_{i_{1}} \cdots s_{i_{j}}$. The expression $h_{\mu}\left(\left(1-q^{-1}\right) X\right)$ on the right-hand side means $\prod_{i=1}^{k+1} h_{\mu_{i}}\left(\left(1-q^{-1}\right) X\right)$, where $h_{m}\left(\left(1-q^{-1}\right) X\right)$ is the symmetric polynomial, homogeneous of degree $m$ in $X$, defined by

$$
\sum_{m \geq 0} h_{m}\left(\left(1-q^{-1}\right) X\right)=\prod_{i} \frac{1-q^{-1} x_{i}}{1-x_{i}} .
$$

Combining (4.5) and (4.6) leads to the generating function

$$
\begin{aligned}
1+\sum_{n \geq 1} \psi\left(q^{(n-1) / 2} C_{w_{n}}^{\prime}\right) & =\frac{1}{1-\frac{1}{q-1} \sum_{k \geq 1} q^{k} h_{k}\left(\left(1-q^{-1}\right) X\right)} \\
& =\frac{1}{1-\frac{1}{q-1}(H(q X) / H(X)-1)} \\
& =\frac{H(X)}{H(X)-\frac{1}{q-1}(H(q X)-H(X))},
\end{aligned}
$$

where $H(X)=\sum_{m} h_{m}(X)=\prod_{i}\left(1-x_{i}\right)^{-1}$. The final formula in (4.8) is just another way of writing (4.4), so the proof is complete.

Proposition 4.2 establishes Conjecture 2.1 for $w=w_{n}$. To see this, rewrite (4.4) as

$$
1+\frac{\sum_{n \geq 1}\left(1+q+\cdots+q^{n-1}\right) h_{n}(X)}{1-\sum_{n \geq 2}\left(q+q^{2}+\cdots+q^{n-1}\right) h_{n}(X)} .
$$

In this form it is easy to see that the coefficient of $h_{\lambda}$ is a polynomial in $q$ with nonnegative coefficients, unimodal and symmetric about $q^{(|\lambda|-1) / 2}$.

The generating function (4.4) is an interesting one which has arisen in other contexts $[5,28,31]$. Specifically, as explained in [28], associated with the Coxeter complex of $S_{n}$ is a smooth toric variety whose homology groups carry an 
$S_{n}$ action. Let $P_{\lambda}(q)$ be the polynomial whose coefficients are the multiplicities of $V_{\lambda}$ in these homology groups. Then $\sum_{\lambda} P_{\lambda}(q) s_{\lambda}$ turns out to be given by (4.4). In other words,

$$
P_{\lambda}(q)=\chi_{\lambda}\left(q^{\ell\left(w_{n}\right) / 2} C_{w_{n}}^{\prime}\right) .
$$

No direct explanation of (4.10) seems to be known, nor whether the corresponding result holds for every Coxeter group. It is known that $\sum_{\lambda} f_{\lambda} P_{\lambda}(q)=$ $\sum_{w \in W} q^{|D(w)|}$ for every Coxeter group $W$, where $|D(w)|$ is the number of descents of $w$, i.e., the number of simple reflections $s$ such that $s w<w$. This follows from the fact that there is a $W$-equivariant isomorphism between the homology of the toric variety and a suitable quotient of the Stanley-Reisner ring of the Coxeter complex. It is not hard to show that (4.10) agrees with this.

We close this section with a conjectured exact value for certain $\phi_{\lambda}(w)$. Conjecture 4.1. Let $w_{f}$ be codominant ( $f$ as in Proposition 3.1). Then

$$
\phi_{(n)}\left(q^{\ell\left(w_{f}\right) / 2} C_{w_{f}}^{\prime}\right)=[n]_{q}[f(1)-1]_{q}[f(2)-2]_{q} \cdots[f(n-1)-(n-1)]_{q},
$$

where $[k]_{q}=1+q+\cdots+q^{k-1}$.

This conjecture agrees with Propositions 4.1 and 4.2 in the cases where they overlap. Its $q=1$ specialization will be proven in the next section.

\section{THE CASE $q=1$}

The case of Conjecture 2.1 for $q=1$ and $w=w_{f}$ codominant is equivalent to a special case of Conjecture 1.4. To see this, choose $\mu$ and $\nu$ such that the 0 entries of $H_{\mu / \nu}$ form the forbidden pattern describing permutations $v \leq w_{f}$ (such $\mu$ and $\nu$ can always be found). Then the term $T_{v} h_{\mu-v(\nu)}$ in (2.1) is nonzero exactly for $v \leq w_{f}$, and the coefficient of $s_{(N)}$ in $I_{\mu / \nu}$ is $C_{w_{f}}^{\prime}(1)$, where $N=|\mu|-|\nu|$. Hence Conjecture 1.4 implies Conjecture 2.1.

Stanley and Stembridge [29] proved Conjecture 1.4 in two cases. First, they proved it when $\mu / \nu$ is a 'rim hook', corresponding to the $n$-cycle $w_{f}=$ $\left(\begin{array}{llll}1 & 2 & \cdots & n\end{array}\right)$. Their result in this case is equivalent to the $q=1$ specialization of Proposition 4.2.

Second, they proved the part we need concerning the coefficient of $s_{(N)}$ in the case that the forbidden pattern is not too big, which leads to the following corollary.

Proposition 5.1 [29]. Let $w_{f}$ be codominant, let $k$ be the least value for which $f(k)=n$, and assume $f(1) \geq k-1$. Then $\phi_{\lambda}\left(C_{w_{f}}^{\prime}(1)\right) \geq 0$ for all $\lambda$.

Conjecture 4.1 can also be proven for $q=1$, as follows.

Proposition 5.2. Let $w_{f}$ be codominant. Then

$$
\phi_{(n)}\left(C_{w_{f}}^{\prime}(1)\right)=n(f(1)-1) \cdots(f(n-1)-(n-1)) .
$$


Proof. Let $V$ be the pattern avoided by ones in permutation matrices corresponding to $v \leq w_{f}$. This pattern $V$ consists of the last $n-f(i)$ entries in column $i$ for each $i$. On $S_{n}$, the monomial character $\phi_{(n)}(w)$ is $n$ if $w$ is an $n$-cycle and 0 otherwise. What (5.1) asserts, therefore, is that the number of $n$-cycles whose permutation matrix avoids $V$ is given by $(f(1)-1)(f(2)-2) \cdots(f(n-1)-(n-1))$.

Let $r_{k}$ be the number of ways to place $k$ mutually nonattacking rooks on a chessboard of shape $V$, that is, the number of subsets of $V$ in which no two elements share a row or a column. Consider a fixed such 'rook placement'. Permutations in $S_{n}$ whose matrix is 1 at the positions of all the rooks correspond in a fairly obvious bijective way to permutations in $S_{n-k}$, with $n$-cycles corresponding to $(n-k)$-cycles. It follows by the sieve principle that the number of $n$-cycles avoiding $V$ is $\sum_{k}(-1)^{k} r_{k}(n-k-1)$ !.

By the sieve principle we also find that the total number of permutations avoiding $V$ is $\sum_{k}(-1)^{k} r_{k}(n-k)$ !. Thus the number of $n$-cycles avoiding $V$ is the same as the number of all permutations avoiding a pattern of the same shape as $V$ in an $(n-1) \times(n-1)$ matrix, which is easily seen to be $(f(1)-1)(f(2)-2) \cdots(f(n-1)-(n-1))$.

\section{THE CASE $q=0$}

In this section we calculate $\phi_{\lambda}\left(q^{\ell(w) / 2} C_{w}^{\prime}\right)$ for all $\lambda$ and $w$, with $q=0$. The calculation confirms Conjectures 2.1,3.1, and 4.1 for the specialization $q=0$.

The Kazhdan-Lusztig basis elements $C_{w}^{\prime}$ are not well defined for $q=0$, but $q^{\ell(w) / 2} C_{w}^{\prime}$ is well defined and equal to

$$
C_{w}^{0}=\sum_{v \leq w} T_{v}
$$

since $P_{v, w}(0)=1$ for all $v \leq w$.

The irreducible and monomial 'characters' are not properly named for $q=0$, since $H_{n}(0)$ is not a semisimple algebra. Nevertheless they are well defined by specialization from $H_{n}(q)$, and in particular they may be computed from the $W$-graph form of the cell representations by setting $q=0$.

Proposition 6.1. The monomial characters $\phi_{\lambda}$ of $H_{n}(0)$ take the values

$$
\phi_{\lambda}\left(C_{w}^{0}\right)= \begin{cases}1, & W_{J(w)} \cong S_{\lambda}, \\ 0, & \text { otherwise. }\end{cases}
$$

Here $J(w)$ is the set of simple reflections appearing in a reduced expression for $w, W_{J(w)}$ is the corresponding parabolic subgroup of $S_{n}$ (the smallest parabolic subgroup containing $w)$, and $W_{J(w)} \cong S_{\lambda}$ means $W_{J(w)}$ is a subgroup of the form $S_{\lambda_{1}} \times \cdots \times S_{\lambda_{k}}$ with the factors in any order.

Remark. Conjecture 2.1 requires that $\phi_{\lambda}\left(C_{w}^{0}\right)=1$ for one $\lambda$ and $\phi_{\lambda}\left(C_{w}^{0}\right)=0$ for all others, since by (2.1) the sum over all $\lambda$ is 1 . Conjecture 4.1 , taken 
together with Proposition 4.1, requires that for codominant $w$, the $\lambda$ with $\phi_{\lambda}\left(C_{w}^{0}\right)=1$ is the one given by Proposition 6.1. This is compatible with Conjecture 3.1 and indicates that the leading codominant $w_{1}$ in the expansion of $C_{w}^{\prime}$ should have $J\left(w_{1}\right)=J(w)$.

We need a simple lemma for the proof of Proposition 6.1.

Lemma 6.1. Let $I$ be a set of simple reflections such that $I \cap J(w) \neq \emptyset$. Then

$$
\sum_{\substack{v \leq w \\ v \in W_{I}}}(-1)^{\ell(v)}=0
$$

Proof. Since $w$ cannot be 1 , the left descent set $D(w)$ is nonempty. Let $t \in D(w)$. If $t \in I$, then $v \leq w \Leftrightarrow t v \leq w$ and $v \in W_{I} \Leftrightarrow t v \in W_{I}$. This implies (6.3).

If $t \notin I$, we proceed by induction on $\ell(w)$. We have $\ell(t w)<\ell(w)$ and $J(w) \subseteq J(t w) \cup\{t\}$, which implies $J(t w) \cap I \neq \emptyset$. By induction, the sum of $(-1)^{\ell(v)}$ over $W_{I} \cap\{v \mid v \leq t w\}$ is zero. But $W_{I} \cap\{v \mid v \leq t w\}=W_{I} \cap\{v \mid$ $v \leq w\}$, for if we fix a reduced expression $t U$ for $w$ then either set consists of all $v$ having reduced expressions which use only simple reflections in $I$ and are subwords of $U$.

Proof of Proposition 6.1. By Möbius inversion on the Bruhat order,

$$
T_{w}=\sum_{v \leq w}(-1)^{\ell(v w)} C_{w}^{0}
$$

Hence the proposition is equivalent to

$$
\phi_{\lambda}\left(T_{w}\right)=\sum_{\substack{v \leq w \\ W_{J(v)} \cong S_{\lambda}}}(-1)^{\ell(v w)}
$$

or

$$
\chi_{\lambda}\left(T_{w}\right)=\sum_{\mu} K_{\lambda, \mu} \sum_{\substack{v \leq w \\ W_{J(v)} \cong S_{\mu}}}(-1)^{\ell(v w)}=\sum_{v \leq w}(-1)^{\ell(v w)} K_{\lambda, \mu(v)},
$$

where $\mu(v)$ is the type of the parabolic subgroup $W_{J(v)}$, and $s_{\lambda}=\sum_{\mu} K_{\lambda, \mu} m_{\mu}$.

The 'Kostka number' $K_{\lambda, \mu(v)}=\left\langle s_{\lambda}, h_{\mu(v)}\right\rangle$ has a combinatorial description: it is the number of standard Young tableaux $T$ of shape $\lambda$ whose descent set $D(T)$ is disjoint from $J(v)$. Hence the last expression in (6.6) is equal to

$$
\begin{aligned}
\sum_{T \in \mathrm{SYT}(\lambda)} \sum_{\substack{v \leq w \\
v \in W_{S \backslash D(T)}}}(-1)^{\ell(v w)} & =(-1)^{\ell(w)}|\{T \in \mathrm{SYT}(\lambda) \mid D(T) \supseteq J(w)\}| \\
& =(-1)^{\ell(w)} K_{\lambda^{\prime}, \mu(w)}
\end{aligned}
$$

by Lemma 6.1 and the fact that the inner sum has only the term $v=1$ for $D(T) \supseteq J(w) . \quad \operatorname{SYT}(\lambda)$ stands for the set of standard Young tableaux of shape $\lambda$. 
Now we prove $\chi_{\lambda}\left(T_{w}\right)=(-1)^{\ell(w)} K_{\lambda^{\prime}, \mu(w)}$ for $q=0$. Recall that the cell representation $V_{\lambda}$ can be regarded as spanned by the vertices of a $W$-graph $G_{\lambda}$. These vertices are indexed by tableaux $T \in \operatorname{SYT}(\lambda)$, and each is marked with the descent set $D(T)$. The edges are labelled with certain integers $\widetilde{\mu}(S, T)$ which are irrelevant for us.

The element $T_{s} \in H_{n}(q)$ acts on $V_{\lambda}$ as follows. If $s \in D(T)$, then $T_{s}(T)=$ $-T+q^{1 / 2} \sum_{s \notin D(S)} \widetilde{\mu}(S, T) S$. If $s \notin D(T)$, then $T_{s}(T)=q T$. Thus, for $q=0$, the matrix of $T_{w}$ is diagonal, with $(T, T)$ entry $(-1)^{\ell(w)}$ if $w \in W_{D(T)}$ and zero otherwise. It is obvious that the trace of this matrix is given by (6.7).

\section{Proof of the main theorem of Section 1}

In this section we prove Theorem 1.5, using a generalization of the Steinberg tensor product theorem to infinite-dimensional irreducible highest weight modules over a simple complex Lie algebra (Proposition 7.2). The derivation of this generalized Steinberg theorem is parallel to the usual derivation in the finitedimensional case (see, e.g., [11]), with the role of the Weyl character formula played by the Kazhdan-Lusztig conjecture.

We require the following notation. Let $\mathfrak{g}$ be a finite-dimensional simple complex Lie algebra with Weyl group $W$ (for our application, $\mathfrak{g}=\mathfrak{s l}_{n}$ and $W=S_{n}$ ). As usual, $\delta$ denotes one-half the sum of the positive roots. Let $M_{\lambda}$ denote the Verma module with highest weight $\lambda, L_{\lambda}$ the corresponding irreducible module (infinite-dimensional for nondominant $\lambda$ ).

Because we must consider tensor products of infinite-dimensional modules, we shall work with a class of $\mathfrak{g}$-modules somewhat larger than the commonly used Bernstein-Gel' fand-Gel' fand category $\mathscr{O}$. Thus we define the class $\mathscr{C}$ to contain a $\mathfrak{g}$-module $V$ if:

(1) $V$ is a direct sum $V=\bigoplus_{\lambda} V_{\lambda}$ of weight spaces belonging to integral weights $\lambda$.

(2) For every integer $N, \bigoplus_{\langle\lambda, \delta\rangle \geq N} V_{\lambda}$ is finite dimensional.

The class $\mathscr{C}$ contains the Verma modules $M_{\lambda}$ and the irreducible modules $L_{\lambda}$. The formal character of $V \in \mathscr{C}$ we define by $\operatorname{ch}(V)=\sum_{\lambda} \operatorname{dim}\left(V_{\lambda}\right) x^{\lambda}$, where the symbols $x^{\lambda}$ denote formal exponentials, multiplying by the rule $x^{\lambda} x^{\mu}=x^{\lambda+\mu}$.

Lemma 7.1. Let $W \subseteq V$ be a submodule of $V \in \mathscr{C}$. Then $W$ and $V / W$ belong to $\mathscr{C}$, and we have $\operatorname{ch}(V)=\operatorname{ch}(W)+\operatorname{ch}(V / W)$. If in addition $U \in \mathscr{C}$, then $U \otimes V \in \mathscr{C}$, and we have $\operatorname{ch}(U \otimes V)=\operatorname{ch}(U) \operatorname{ch}(V)$.

Proof. For the first part, everything is clear, provided we know that $W$ is the direct sum of its weight spaces. But every element $a \in W$ belongs to $V$, and hence $a \in V_{\lambda_{1}} \oplus \cdots \oplus V_{\lambda_{k}}$ for a finite set of weights $\left\{\lambda_{i}\right\}$. This space is a finitedimensional module for the Cartan subalgebra $\mathfrak{h} \subseteq \mathfrak{g}$, and so is its intersection with $W$. Since every such module is a direct sum of weight spaces, each weight space component of $a$ is again in $W$, so $W=\bigoplus_{\lambda} W_{\lambda}$. 
For the second part, if we let $\left\{a_{\alpha}\right\}$ and $\left\{b_{\beta}\right\}$ be bases of weight vectors for $U$ and $V$ respectively, then $\left\{a_{\alpha} \otimes b_{\beta}\right\}$ is a basis of weight vectors for $U \otimes V$. The properties asserted for $U \otimes V$ follow easily.

Modules in $\mathscr{C}$ (even in $\mathscr{O}$ ) are not necessarily direct sums of irreducibles, but for formal computations with multiplicities it suffices to use the weaker notion of 'composition series' defined as follows.

Definition. A composition series of a module $V \in \mathscr{C}$ is a finite or infinite sequence

$$
0=W_{0} \subseteq W_{1} \subseteq W_{2} \subseteq \cdots
$$

of submodules $W_{i} \subseteq V$, with $\bigcup_{i} W_{i}=V$ and $W_{i+1} / W_{i}$ an irreducible highest weight module $L_{\lambda_{i}}$ for each $i$.

Note that the weight spaces $\left(W_{i}\right)_{\lambda}$ form a filtration of the finite-dimensional space $V_{\lambda}$, so only a finite number of the quotients $\left(W_{i+1}\right)_{\lambda} /\left(W_{i}\right)_{\lambda}$ are nonzero for any given $\lambda$. Thus $\sum_{i=0}^{\infty} \operatorname{ch}\left(W_{i+1} / W_{i}\right)$ is well defined and equal to $\operatorname{ch}(V)$.

Lemma 7.2. Every $V \in \mathscr{C}$ has a composition series. The number $m_{\lambda}(V)$ of indices $i$ for which $W_{i} / W_{i+1} \cong L_{\lambda}$ is finite and uniquely determined by the equation

$$
\sum_{\lambda} m_{\lambda}(V) \operatorname{ch}\left(L_{\lambda}\right)=\operatorname{ch}(V) .
$$

In particular, $m_{\lambda}(V)$ does not depend on the choice of composition series.

Proof. Obviously we may assume $V \neq 0$.

Let $\lambda$ be a weight for which $V_{\lambda} \neq 0$ and $\langle\lambda, \delta\rangle$ is maximal. Such a weight clearly exists for $V \in \mathscr{C}$. Choose a nonzero $a \in V_{\lambda}$, and let $V_{a}$ be the submodule generated by $a$. Since $a$ is a highest weight vector, $V_{a}$ is a quotient of the Verma module $M_{\lambda}$ and therefore has a finite composition series

$$
0=U_{0} \subseteq U_{1} \subseteq \cdots \subseteq U_{i}=V_{a} .
$$

If we have constructed a partial composition series

$$
0=W_{0} \subseteq W_{1} \subseteq \cdots \subseteq W_{k} \neq V
$$

for $V$, we may apply the above construction to a maximal weight vector $a$ in $V / W_{k}$ to obtain an extension

$$
0=W_{0} \subseteq W_{1} \subseteq \cdots \subseteq W_{k} \subseteq \cdots \subseteq W_{l}=W_{k}+V_{a} .
$$

Repeating the process, we either reach $W_{l}=V$ at some stage, or we produce an infinite sequence

$$
0=W_{0} \subseteq W_{1} \subseteq \cdots
$$

At each stage, we reduce the dimension of a nonzero weight space $\left(V / W_{l}\right)_{\lambda}$ for which $\langle\lambda, \delta\rangle$ is maximal. By condition (2) in the definition of $\mathscr{C}$, we must 
eventually exhaust every weight space, i.e., $\left(V / W_{l}\right)_{\lambda}=0$ for sufficiently large $l$. Hence our sequence is a composition series.

As for the multiplicities, clearly $L_{\lambda}$ cannot occur as a quotient $W_{i+1} / W_{i}$ more than $\operatorname{dim}\left(V_{\lambda}\right)$ times, so $m_{\lambda}(V)$ is finite. Equation (7.2) is thus well defined and valid. The formal characters $\operatorname{ch}\left(L_{\lambda}\right)$ are linearly independent, since each has a distinct leading term $x^{\lambda}$. Therefore, (7.2) determines the multiplicities $m_{\lambda}(V)$.

Remark. The rest of our work in this section consists entirely of computations upon formal power series $f(x)=\sum_{\lambda} c_{\lambda} x^{\lambda}$ with integer coefficients obeying

$\left(2^{\prime}\right)$ For every integer $N$, there are only finitely many weights $\lambda$ with $\langle\lambda, \delta\rangle \geq N$ and $c_{\lambda} \neq 0$.

Formal series obeying $\left(2^{\prime}\right)$ form a ring, and they have unique expansions $f(x)=$ $\sum_{\lambda} d_{\lambda} \operatorname{ch}\left(M_{\lambda}\right)=\sum_{\lambda} e_{\lambda} \operatorname{ch}\left(L_{\lambda}\right)$, where the coefficients $d_{\lambda}$ and $e_{\lambda}$ obey the same restriction $\left(2^{\prime}\right)$ as $c_{\lambda}$. The formal series $f(x)$ is actually the formal character of some module in $\mathscr{C}$ if and only if the coefficients $e_{\lambda}$ are nonnegative. Our need for the underlying representation theory is confined to a single consequence of it: the product of two series which are actually characters is again a character.

We now proceed to the derivation of the generalized Steinberg tensor product theorem from the Kazhdan-Lusztig conjecture.

It will be convenient to define some notation in connection with the action of $W$ on weights. If $\lambda$ is a weight and $w$ is an element of $W$, define $w \cdot \lambda=$ $w(\lambda+\delta)-\delta$. If $\lambda+\delta$ is dominant, then $W^{\lambda}=\{w \mid w \cdot \lambda=\lambda\}$ denotes the stabilizer of $\lambda+\delta$, a parabolic subgroup of $W$. Each coset $w W^{\lambda}$ contains a unique longest element; let $D^{\lambda}$ denote the set of these.

Definition. The expression $w \cdot \lambda$ is in standard form if: (1) $\lambda+\delta$ is dominant, and (2) $w \in D^{\lambda}$.

Every weight has a unique expression in standard form.

The formal characters of Verma modules are given by

$$
\operatorname{ch}\left(M_{\lambda}\right)=x^{\lambda} / \Delta(x), \quad \text { where } \Delta(x)=\prod_{\alpha}\left(1-x^{-\alpha}\right),
$$

the product taken over all positive roots $\alpha$.

By Harish-Chandra's theorem, $M_{v \cdot \lambda}$ has a finite composition series whose composition factors are of the form $L_{w \cdot \lambda}$. Their multiplicities are given by the Kazhdan-Lusztig conjecture:

Proposition $7.1([2,6,12])$. For $\lambda+\delta$ dominant and integral we have

$$
\operatorname{ch}\left(M_{v \cdot \lambda}\right)=\sum_{w \in D^{\lambda}} P_{v, w}(1) \operatorname{ch}\left(L_{w \cdot \lambda}\right)
$$


or equivalently

$$
\sum_{w \in W}(-1)^{\ell(v w)} Q_{v, w}(1) \operatorname{ch}\left(M_{w \cdot \lambda}\right)= \begin{cases}\operatorname{ch}\left(L_{v \cdot \lambda}\right), & v \in D^{\lambda}, \\ 0, & v \notin D^{\lambda},\end{cases}
$$

where $P_{v, w}$ and $Q_{v, w}=P_{w_{0} w, w_{0} v}$ are Kazhdan-Lusztig polynomials for $W$.

The proofs in [2] and [6] treat the case $\lambda=0$. The reduction of the general case to this one had already been found in [12]. The equivalence of (7.4) with (7.5) is a consequence of the fact that $\left[P_{v, w}\right]$ and $\left[(-1)^{\ell(v w)} Q_{v, w}\right]$ are inverse $|W| \times|W|$ matrices.

Proposition 7.2 (Generalized Steinberg theorem). Let $s \cdot \alpha, t \cdot \beta$, and $u \cdot \gamma$ be integral weights expressed in standard form. Then the multiplicity of $L_{u \cdot \gamma}$ as a composition factor of $L_{s \cdot \alpha} \otimes L_{t \cdot \beta}$ is given by

$$
\sum_{\substack{r \in D^{\gamma} \\ v, w \in W}}(-1)^{\ell(s t v w)} P_{r, u}(1) Q_{s, v}(1) Q_{t, w}(1) \mathfrak{p}(v \cdot \alpha+w \cdot \beta-r \cdot \gamma),
$$

where $\mathfrak{p}(\lambda)$ is the partition function, defined by

$$
\frac{1}{\Delta(x)}=\sum_{\lambda} \mathfrak{p}(\lambda) x^{-\lambda}
$$

Proof. If $f$ is a formal character, let $\left\langle\operatorname{ch}\left(L_{\lambda}\right)\right\rangle f$ denote the coefficient of $\operatorname{ch}\left(L_{\lambda}\right)$ in the expansion of $f$ by irreducible characters, and let $\left\langle\operatorname{ch}\left(M_{\lambda}\right)\right\rangle f$ denote the coefficient of $\operatorname{ch}\left(M_{\lambda}\right)$ in its expansion by Verma module characters. By (7.4) we have, for $u \cdot \gamma$ standard,

$$
\left\langle\operatorname{ch}\left(L_{u \cdot \gamma}\right)\right\rangle f=\sum_{r \in D^{\gamma}} P_{r, u}(1)\left\langle\operatorname{ch}\left(M_{r \cdot \gamma}\right)\right\rangle f .
$$

We are to show that $\left\langle\operatorname{ch}\left(L_{u \cdot \gamma}\right)\right\rangle \operatorname{ch}\left(L_{s \cdot \alpha}\right) \operatorname{ch}\left(L_{t \cdot \beta}\right)$ is equal to (7.6). By (7.5),

$$
\operatorname{ch}\left(L_{s \cdot \alpha}\right) \operatorname{ch}\left(L_{t \cdot \beta}\right)=\sum_{v, w \in W}(-1)^{\ell(s t v w)} Q_{s, v}(1) Q_{t, w}(1) \operatorname{ch}\left(M_{v \cdot \alpha}\right) \operatorname{ch}\left(M_{w \cdot \beta}\right) .
$$

Note that $\left\langle\operatorname{ch}\left(M_{r \cdot \gamma}\right)\right\rangle \operatorname{ch}\left(M_{v \cdot \alpha}\right) \operatorname{ch}\left(M_{w \cdot \beta}\right)=\left\langle x^{r \cdot \gamma}\right\rangle\left(x^{v \cdot \alpha+w \cdot \beta} / \Delta(x)\right)$, by (7.3). Applying this to (7.8) with $f$ given by (7.9) yields

$$
\begin{aligned}
\left\langle\operatorname{ch}\left(L_{u \cdot \gamma}\right)\right\rangle \operatorname{ch}\left(L_{s \cdot \alpha}\right) \operatorname{ch}\left(L_{t \cdot \beta}\right) & \\
\quad= & \sum_{\substack{r \in D^{\gamma} \\
v, w \in W}}(-1)^{\ell(s t v w)} P_{r, u}(1) Q_{s, v}(1) Q_{t, w}(1)\left\langle x^{r \cdot \gamma}\right\rangle\left(x^{v \cdot \alpha+w \cdot \beta} / \Delta(x)\right) \\
\quad & \sum_{\substack{r \in D^{\gamma} \\
v, w \in W}}(-1)^{\ell(s t v w)} P_{r, u}(1) Q_{s, v}(1) Q_{t, w}(1) \mathfrak{p}(v \cdot \alpha+w \cdot \beta-r \cdot \gamma) .
\end{aligned}
$$


Observe that (7.6) reduces to zero when $s \notin D^{\alpha}$ or $t \notin D^{\beta}$. This is clear from the proof, since (7.9) reduces to zero in these cases, by (7.5).

Finally, we apply this to immanants.

Proof of Theorem 1.5. The coefficient of $C_{t}^{\prime}(1) s_{\kappa}(X)$ in $I_{\mu / \nu}$ is given by

$$
m_{\mu / \nu}^{t, \kappa}=\sum_{w \in S_{n}}(-1)^{\ell(t w)} Q_{t, w}(1) K_{\kappa, \mu-w(\nu)},
$$

where the Kostka number $K_{\kappa, \lambda}$ is by definition the coefficient of $s_{\kappa}$ in $h_{\lambda}$. It is well known that

$$
K_{\kappa, \lambda}=\sum_{v \in S_{n}}(-1)^{\ell(v)} \mathfrak{p}(v \cdot \kappa-\lambda),
$$

where $\kappa$ is a partition, regarded as a dominant weight for $\mathfrak{s l}_{n}$, and $\lambda$ is any sequence $\left(\lambda_{1}, \ldots, \lambda_{n}\right) \in \mathbb{Z}^{n}$ with $|\lambda|=|\kappa|$. Indeed, (7.12) is equivalent to the fact that $\operatorname{ch}\left(L_{\kappa}\right)$ is the Schur function $s_{\kappa}$ for $\kappa$ dominant.

Combining (7.11) and (7.12) we obtain

$$
m_{\mu / \nu}^{t, \kappa}=\sum_{v, w \in S_{n}}(-1)^{\ell(t v w)} Q_{t, w}(1) \mathfrak{p}(v \cdot \kappa+w \cdot(\nu-\delta)-(\mu-\delta))
$$

By Proposition 7.2, this is a nonnegative integer, since it is (7.6) with $\alpha=\kappa$, $s=1, \beta=\nu-\delta, t=t, \gamma=\mu-\delta$, and $u \in D^{\gamma}$ representing the coset $1 W^{\gamma}$. Note that $\alpha+\delta, \beta+\delta$, and $\gamma+\delta$ are dominant as required, since they are partitions, and $u \cdot \gamma$ and $s \cdot \kappa$ are in standard form. For $t \notin D^{\beta}, t \cdot \beta$ is not standard, but then, as we observed above, (7.6) reduces to zero.

It is instructive to examine the case $t=1$. Extracting the coefficient of $C_{1}^{\prime}(1) s_{\kappa}(X)$ in $I_{\mu / \nu}$ amounts to finding the coefficient of $s_{\kappa}(X)$ in $\operatorname{det}\left(H_{\mu / \nu}\right)=$ $\operatorname{det}\left(H_{\gamma+\delta / \beta+\delta}\right)=s_{\gamma / \beta}(X)$. The equality of this with $\left\langle s_{\gamma}, s_{\kappa} s_{\beta}\right\rangle$ is the defining identity for skew Schur functions. But, of course, $\left\langle s_{\gamma}, s_{\kappa} s_{\beta}\right\rangle$ is the multiplicity of $L_{\gamma}$ in $L_{\kappa} \otimes L_{\beta}$, since $\operatorname{ch}\left(L_{\lambda}\right)=s_{\lambda}$ for dominant $\lambda$. Thus the symmetric functions $\left\langle C_{w}^{\prime}(1)\right\rangle I_{\gamma+\delta / \beta+\delta}$ appear as rather natural generalizations of the skew Schur function $s_{\gamma / \beta}$.

It may also be worth noting that the coefficient of $s_{\kappa}$ in $\left\langle C_{w}^{\prime}(1)\right\rangle I_{\gamma+\delta / \beta+\delta}$ is the multiplicity of $L_{\gamma}$ in $L_{\kappa} \otimes L_{w \cdot \beta}$ for $w \cdot \beta$ standard and zero otherwise. Since $L_{\kappa}$ is finite dimensional, this particular tensor product does belong to the category $\mathscr{O}$, not just to $\mathscr{C}$.

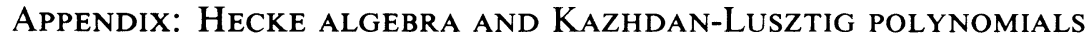

Here we state the definitions and theorems we use from Kazhdan-Lusztig theory. Since this paper is meant for a varied audience, this material has been included for completeness, despite the risk that it may be unnecessary to specialists and incomprehensible to everyone else. Standard references are [4] and [17]. 
The symmetric group $S_{n}$ is an example of a Coxeter group, with its distinguished generating set of simple reflections $S$ consisting of the adjacent transpositions $(i i+1)$. A minimum-length expression for $w \in S_{n}$ as a product of simple reflections is called a reduced expression; its length $\ell(w)$ is the number of inversions in $w$. The sign of $w$ is $(-1)^{\ell(w)}$.

The Bruhat order is a partial order on $S_{n}$ with the defining property that $v \leq w$ if for some, or equivalently for every, reduced expression $s_{1} \cdots s_{\ell}$ for $w$, there is a subword $s_{i_{1}} \cdots s_{i_{k}}$ which is a reduced expression for $v$. The Bruhat order is the transitive closure of the relation $v \prec w$ if $v=w \cdot(i j)$ for some $i<j$ such that $w(i)>w(j)$.

For each $s \in S$, either $s w>w$ or $s w<w$ in Bruhat order. Those $s$ with $s w<w$ are the (left) descents of $w$. The descent set is denoted $D(w)$. There is a reduced expression for $w$ beginning with $s$ if and only if $s \in D(w)$.

A subgroup $W_{J} \subseteq S_{n}$ generated by a subset $J \subseteq S$ is called parabolic. If $\left(i_{1} i_{1}+1\right),\left(i_{2} i_{2}+1\right), \ldots,\left(i_{k} i_{k}+1\right)$ are the simple reflections not in $J$, with $i_{1}<\cdots<i_{k}$, then $W_{J}$ has the form $S_{\lambda_{1}} \times \cdots \times S_{\lambda_{k+1}}$, where $\lambda=$ $\left(i_{1}, i_{2}-i_{1}, \ldots, n-i_{k}\right)$. Each coset $w W_{J}$ contains a unique longest and Bruhat greatest element, which can be obtained by sorting each of the successive segments of length $\lambda_{1}, \lambda_{2}, \ldots, \lambda_{k+1}$ in the sequence $w(1), \ldots, w(n)$ into decreasing order. The longest element of $S_{n}$ is denoted $w_{0}$.

A presentation of $S_{n}$ is given by the generators $S$ and relations

$$
\begin{gathered}
s^{2}=1, \\
r s=s r \quad \text { if }|i-j|>1, \\
r s r=s r s \quad \text { if }|i-j|=1,
\end{gathered}
$$

where $r=(i i+1)$ and $s=(j j+1)$. The Hecke algebra $H_{n}(q)$ associated to $S_{n}$ is the algebra with unit over $\mathbb{C}\left[q^{-1 / 2}, q^{1 / 2}\right]$ generated by elements $T_{s}$ for $s \in S$, subject to the relations

$$
\begin{array}{clrl}
T_{r} T_{s} & =T_{s} T_{r} & & \text { if }|i-j|>1, \\
T_{r} T_{s} T_{r} & =T_{s} T_{r} T_{s} & & \text { if }|i-j|=1 .
\end{array}
$$

For $w \in S_{n}$, we define

$$
T_{w}=T_{s_{1}} \cdots T_{s_{\ell}},
$$

where $s_{1} \cdots s_{\ell}$ is a reduced expression for $w$. This is well defined because all reduced expressions for $w$ are connected by relations of the form $(8.1 \mathrm{~b})$. The elements $T_{w}$ form a basis for the Hecke algebra and we have

$$
T_{s} T_{w}= \begin{cases}T_{s w}, & s w>w \\ (q-1) T_{w}+q T_{s w}, & s w<w\end{cases}
$$


In view of $(8.1)$, the specialization $H_{n}(1)$ is the group algebra $\mathbb{C} S_{n}$, with $\left\{T_{w}\right\}$ as the natural basis. For $q$ an indeterminate, $H_{n}(q)$ is a semisimple algebra. Specializing them at $q=1$ sets up a natural correspondence between its irreducible representations and those of $S_{n}$.

There is a $\mathbb{C}$-linear involution of $H_{n}(q)$ defined by

$$
\bar{q}=q^{-1}, \quad \overline{T_{w}}=\left(T_{w^{-1}}\right)^{-1} .
$$

The Kazhdan-Lusztig basis $\left\{C_{w}^{\prime}\right\}$ of $H_{n}(q)$ is uniquely defined by the conditions:

$\overline{C_{w}^{\prime}}=C_{w}^{\prime}, \quad q^{\ell(w) / 2} C_{w}^{\prime}=\sum_{v \leq w} P_{v, w}(q) T_{v}$,

$P_{v, w}(q) \in \mathbb{Z}[q], \quad P_{w, w}(q)=1, \quad \operatorname{deg}\left(P_{v, w}\right)<(\ell(w)-\ell(v)) / 2 \quad$ for $v \neq w$.

The $P_{v, w}(q)$ are Kazhdan-Lusztig polynomials. They have constant term $P_{v, w}(0)=1$ for all $v \leq w$. The inverse of $(8.6)$ is

$$
T_{w}=\sum_{v \leq w}(-1)^{\ell(v w)} Q_{v, w}(q) q^{\ell(v) / 2} C_{v}^{\prime},
$$

where $Q_{v, w}=P_{w_{0} w, w_{0} v}$.

The basis $C_{w}^{\prime}$ satisfies the two equivalent formulas

$$
\begin{gathered}
C_{s}^{\prime} C_{w}^{\prime}= \begin{cases}C_{s w}^{\prime}+\sum_{s v<v} \mu(v, w) C_{v}^{\prime}, & s w>w, \\
\left(q^{-1 / 2}+q^{1 / 2}\right) C_{w}^{\prime}, & s w<w ;\end{cases} \\
T_{s} C_{w}^{\prime}= \begin{cases}-C_{w}^{\prime}+q^{1 / 2} \sum_{s v<v} \tilde{\mu}(v, w) C_{v}^{\prime}, & s w>w, \\
q C_{w}^{\prime}, & s w<w,\end{cases}
\end{gathered}
$$

where $\mu(v, w)$ is the coefficient of $q^{(\ell(w)-\ell(v)-1) / 2}$ in $P_{v, w}(q)$ if $\ell(w)-\ell(v)$ is odd, zero otherwise, and $\tilde{\mu}(v, w)$ is $\mu(v, w)$ or $\mu(w, v)$, accordingly as $v<w$ or $w<v$.

A reflexive and transitive relation $\leq$ is called a preorder. To a preorder corresponds an equivalence relation defined by $x \approx y$ if $x \leq y \leq x$. The preorder induces a partial order on $\approx$-equivalence classes.

Preorders $\leq_{\mathrm{L}}$ and $\leq_{\mathrm{R}}$ are defined on $S_{n}$ as follows: $\leq_{\mathrm{L}}\left(\right.$ resp. $\left.\leq_{\mathrm{R}}\right)$ is the weakest relation such that for all $w$ the linear span of $\left\{C_{v}^{\prime} \mid v \leq_{\mathrm{L}} w\right\}$ (resp. $\left.\left\{C_{v}^{\prime} \mid v \leq_{\mathrm{R}} w\right\}\right)$ is a left (resp. right) ideal in $H_{n}(q)$. The transitive closure of $\leq_{\mathrm{L}} \cup \leq_{\mathrm{R}}$ is denoted $\leq_{\mathrm{LR}}$; thus $\left\{C_{v}^{\prime} \mid v \leq_{\mathrm{LR}} w\right\}$ spans a two-sided ideal in $H_{n}(q)$.

The equivalence classes of $\approx_{\mathrm{L}}, \approx_{\mathrm{R}}$, and $\approx_{\mathrm{LR}}$ are the left, right, and twosided cells, respectively. Regarding the span $\mathbb{C}\left[q^{-1 / 2}, q^{1 / 2}\right]\left\{C_{v}^{\prime} \mid v \approx_{L} w\right\}$ as a quotient $\mathbb{C}\left[q^{-1 / 2}, q^{1 / 2}\right]\left\{C_{v}^{\prime} \mid v \leq_{\mathrm{L}} w\right\} / \mathbb{C}\left[q^{-1 / 2}, q^{1 / 2}\right]\left\{C_{v}^{\prime} \mid v<_{\mathrm{L}} w\right\}$ makes it a left $H_{n}(q)$-module, called a cell representation. 
Let $(P(w), Q(w))$ be the pair of standard Young tableaux (of shape $\lambda(w)$ ) corresponding to $w$ under the Schensted correspondence. Then $v \approx_{L} w$ if and only if $Q(v)=Q(w), v \approx_{\mathrm{R}} w$ if and only if $P(v)=P(w)$, and $v \approx_{\mathrm{LR}} w$ if and only if $\lambda(v)=\lambda(w)$. It is convenient to index cells by the transposed tableaux $P(v)^{\prime}, Q(v)^{\prime}$ of shape $\lambda^{\prime}$. With this indexing the left cells indexed by tableaux of shape $\lambda$ afford the irreducible representation $V_{\lambda}$, according to the usual indexing for $S_{n}$ modules.

The ordering $<_{\mathrm{LR}}$ on two-sided cells corresponds to the opposite of the dominance order on their indexing partitions. No direct statement of this fact seems to appear in the literature, but it can be deduced without much difficulty from the results of [1] and [16] on primitive ideals in $U\left(\mathfrak{g l}_{n}\right)$.

Using (8.9), the action of $T_{s}$ in a cell representation can be described by a $W$-graph. The vertices correspond to basis elements $C_{v}^{\prime}$ for $v$ in a left cell, hence to the tableaux $P(v)^{\prime}$ of a fixed shape. Vertices $v, w$ are connected by an edge labelled $\tilde{\mu}(v, w)=\widetilde{\mu}(S, T)$, which depends only on the tableaux $S=P(v)^{\prime}, T=P(w)^{\prime}$. Since $D(v)$ is the complement of the descent set $D\left(P(v)^{\prime}\right)$, we have for $s \in D(T), T_{s}(T)=-T+q^{1 / 2} \sum_{s \notin D(S)} \widetilde{\mu}(S, T) S$, and for $s \notin D(T), T_{s}(T)=q T$.

The elements $C_{w}^{\prime}$ are closely connected with the geometry of the flag variety. Let $V$ be an $n$-dimensional complex vector space. The flag variety $\mathscr{F}_{n}$ consists of all flags $0 \subset F_{1} \subset \cdots \subset F_{n-1} \subset F_{n}=V$ of subspaces with $\operatorname{dim}\left(F_{i}\right)=i$. Fix a flag $F^{0}$ and let $B$ be its stabilizer in $\mathrm{GL}_{n}$, i.e., the Borel subgroup of all upper triangular matrices. Each flag $F=g F^{0}$ corresponds to a coset $g B$ of $B$. We have the Bruhat decomposition

$$
\mathrm{GL}_{n}=\bigcup_{w \in S_{n}} B w B
$$

(Regard $w$ as a permutation matrix.) This induces a decomposition of the flag variety into Schubert cells $\gamma_{w}=\left\{g F^{0} \mid g B \subseteq B w B\right\}$ with dimension $\operatorname{dim}\left(\gamma_{w}\right)=\ell(w)$. Their closures $\Gamma_{w}=\bar{\gamma}_{w}$ are the Schubert varieties. We have $\Gamma_{v} \subseteq \Gamma_{w}$ if and only if $v \leq w$ in Bruhat order.

The flags $F \in \Gamma_{w}$ can be described by Schubert conditions with respect to the base flag $F^{0}$. Namely, $F \in \Gamma_{w}$ if and only if for all $i, j, \operatorname{dim}\left(F_{i} \cap F_{j}^{0}\right) \geq$ $|w(\{1, \ldots, i\}) \cap\{1, \ldots, j\}|$.

Let $I H_{v}^{*}\left(\Gamma_{w}\right)$ be the local intersection homology of $\Gamma_{w}$ at a point of $\Gamma_{v}$. This is nonzero in even (real) dimensions only and satisfies (by [18])

$$
P_{v, w}(q)=\sum_{i} q^{i} \operatorname{dim}\left(I H_{v}^{2 d-2 i}\left(\Gamma_{w}\right)\right) .
$$

In particular, the Kazhdan-Lusztig polynomials have nonnegative coefficients. Moreover, they are equal to 1 for all $v \leq w$ if $\Gamma_{w}$ is smooth.

Consider a variety $\Gamma_{u, v}$ consisting of pairs $\left(F^{1}, F^{2}\right)$ such that $F^{1} \in \Gamma_{u}$, and, with respect to $F^{1}$ as a base flag, $F^{2} \in \Gamma_{v}$. Projecting $\Gamma_{u, v}$ on $F^{2}$ 
induces a decomposition of the intersection homology of $\Gamma_{u, v}$ described by certain polynomials with nonnegative, symmetric, and unimodal coefficients [3]. By [27], these polynomials are the structure coefficients $q^{(\ell(u)+\ell(v)-\ell(w)) / 2} f_{u, v}^{w}(q)$ defined by

$$
C_{u}^{\prime} C_{v}^{\prime}=\sum_{w} f_{u, v}^{w}(q) C_{w}^{\prime}
$$

When $q$ is a power of a prime we may consider the finite 'flag variety' over a field $\mathbb{F}(q)$. If we form a complex vector space by taking this finite set of flags as a basis, then the Hecke algebra $H_{n}(q)$ (specialized to this integer $q$ ) acts on it as the algebra of all operators commuting with the action of $\operatorname{GL}_{n}(q)$. The operator $T_{w} \in H_{n}(q)$ maps a flag $F$ to the sum of all flags $F^{\prime}$ in the Schubert cell $\gamma_{w^{-1}}$, constructed with respect to $F$ as base flag. The trace of this action is therefore $\chi\left(T_{w}\right)=0$ for $w \neq 1, \chi\left(T_{1}\right)=\left|\mathscr{F}_{n}(q)\right|=[n]_{q} !=$ $(1)(1+q) \cdots\left(1+q+\cdots+q^{n-1}\right)$. This character (really a different character for each $q$ ) is the one used in the proof following Conjecture 3.1.

\section{REFERENCES}

1. D. Barbasch and D. Vogan, Primitive ideals and orbital integrals in complex exceptional groups. J. Algebra 80 (1983), 350-382.

2. A. Beilinson and J. Bernstein, Localisation des g-modules. C. R. Acad. Sci. Paris Sér. I Math. 292 (1981), 15-18.

3. A. Beilinson, J. Bernstein, and P. Deligne, Faisceaux pervers. Astérisque 100 (1983).

4. N. Bourbaki, Groupes et algebras de Lie, Chapitres 4, 5, 6, Hermann, Paris, (1968).

5. F. Brenti, Unimodal polynomials arising from symmetric functions, Proc. Amer. Math. Soc. 108 (1990), 1133-1141.

6. J. Brylinski and M. Kashiwara, Kazhdan-Lusztig conjecture and holonomic systems, Invent. Math. 64 (1981), 387-410.

7. P. Freyd, D. Yetter, J. Hoste, W. B. R. Lickorish, K. Millett, and A. Ocneanu, A new polynomial invariant of knots and links. Bull. Amer. Math. Soc. 12 (1985), 239-246.

8. M. Goresky and R. MacPherson, Intersection homology. II, Invent. Math. 72 (1983), 77-129.

9. I. Goulden and D. Jackson, Immanants of combinatorial matrices, J. Algebra 148 (1992), 305-324.

10. C. Greene, Proof of a conjecture on immanants of the Jacobi-Trudi matrix, Linear Algebra Appl. 171 (1992), 65-79.

11. N. Jacobson, Lie algebras, Interscience, New York, 1962.

12. J. Jantzen, Moduln mit einem höchsten Gewicht, Lecture Notes in Math., vol. 750, SpringerVerlag, Berlin, Heidelberg, and New York, 1979.

13. M. Jimbo, A q-analogue of $U(g l(N+1))$, Hecke algebra, and the Yang-Baxter equation, Lett. Math. Physics 11 (1986), 247-252.

14. V. F. R. Jones, Hecke algebra representations of braid groups and link polynomials, Ann. of Math. (2) 126 (1987), 335-388.

15. __ Index for subfactors. Invent. Math., 72 (1983) 1-25.

16. A. Joseph, Towards the Jantzen conjecture. III, Compositio Math. 41 (1981), 23-30.

17. D. Kazhdan and G. Lusztig, Representations of Coxeter groups and Hecke algebras, Invent. Math. 53 (1979), 165-184.

18. _ Schubert varieties and Poincaré duality, Proc. Sympos. Pure Math., vol. 36, Amer. Math. Soc., Providence, RI, 1980, pp. 185-203. 
19. R. C. King and B. G. Wybourne, Characters of Hecke algebras $H_{n}(q)$ of type $A_{n-1}$, J. Physics A 23 (1990), L1193-L1197.

20. A. Lascoux and M.-P. Schützenberger, Schubert polynomials and the LittlewoodRichardson rule, Lett. Math. Phys. 10 (1985), 111-124.

21. E. Lieb, Proofs of some conjectures on permanents, J. Math. Mech. 16 (1966), 127-134.

22. D. E. Littlewood, The theory of group characters, 2nd ed., Oxford Univ. Press, London, 1950.

23. I. G. Macdonald, Symmetric functions and Hall polynomials, Oxford Univ. Press, London, 1979.

24. A. Ram, A Frobenius formula for the characters of the Hecke algebras, Invent. Math. 106 (1991), 461-488.

25. I. Schur, Über endliche Gruppen und Hermitesche Formen, Math. Z. 1 (1918), 184-207.

26. M.-P. Schützenberger, La correspondance de Robinson, Combinatoire et Représentation du Groupe Symétrique-Strasbourg, 1976 (D. Foata, ed.), Lecture Notes in Math., vol. 579, Springer-Verlag, Berlin, Heidelberg, and New York, 1977, pp. 59-113.

27. T. A. Springer, Quelques applications de la cohomologie d'intersection, Sém. Bourbaki $\mathbf{5 8 9}$ (1982).

28. R. P. Stanley, Log-concave and unimodal sequences in algebra, combinatorics, and geometry, Graph Theory and Its Applications: East and West, Ann. N. Y. Acad. Sci. 576 (1989), 500-535.

29. R. P. Stanley and J. Stembridge, On immanants of Jacobi-Trudi matrices and permutations with restricted position, J. Combin. Theory Ser. A (to appear).

30. J. Stembridge, Some conjectures for immanants, Canad. J. Math. 44 (1992), 1079-1099.

31. Eulerian numbers, tableaux, and the Betti numbers of a toric variety, Discrete Math. 99 (1992), 307-320.

32. __ Immanants of totally positive matrices are non-negative, Bull. London Math. Soc. 23 (1991), 422-428.

33. J. Vanderjeugt, An algorithm for characters of Hecke algebras $H_{n}(q)$ of type $A_{n-1}$, J. Physics A 24 (1991), 3719-3725.

34. H. Wenzl, Hecke algebras of type $A_{n}$ and subfactors, Invent. Math. 92 (1988), 349-383.

Department of Mathematics, University of California, San Diego, la Jolla, CaliforNIA 92093-0112

E-mail address: mhaiman@macaulay.ucsd.edu 